02

\title{
Особенности нелинейного отклика структуры сверхпроводник-нормальный металл с большим отношением удельных сопротивлений
}

\author{
(C) Е.Е. Пестов ${ }^{1,2}$, Ю.Н. Ноздрин ${ }^{1}$, В.В. Рогов ${ }^{1}$, И.Ю. Пашенькин ${ }^{1}$, Д.Ю. Водолазов ${ }^{1}$ \\ ${ }^{1}$ Институт фризики микроструктур РАН, \\ Нижний Новгород, Россия \\ ${ }^{2}$ Нижегородский государственный университет им. Н.И. Лобачевского, \\ Нижний Новгород, Россия \\ E-mail: pestov@ipmras.ru
}

Поступила в Редакцию 19 апреля 2019 г.

В окончательной редакции 22 апреля 2019 г.

Принята к публикации 24 апреля 2019 г.

С помощью метода ближнепольной СВЧ микроскопии исследована температурная зависимость мощности третьей гармоники $P_{3 \omega}(T)$ структур сверхпроводник-нормальный металл MoN/Al с эффектом близости. Обнаружено наличие дополнительного (низкотемпературного) максимума и занулений в зависимости $P_{3 \omega}(T)$ для структуры MoN/Al, которые исчезают при наложении слабого, перпендикулярного плоскости структуры, магнитного поля.

Ключевые слова: нелинейность, СВЧ, структура сверхпроводник-нормальный металл, ближнепольная СВЧ микроскопия.

DOI: $10.21883 /$ FTT.2019.09.48094.23N

\section{1. Введение}

Хорошо известно, что в случае частично или полностью прозрачной границы сверхпроводник-нормальный металл $(S N)$ куперовские пары из сверхпроводника могут проникать в нормальный металл на конечное расстояние из-за наличия эффекта близости [1]. В этом случае в пленке нормального металла возникает индуцированная сверхпроводимость, которая принимает участие в экранировании приложенного постоянного или переменного магнитного поля [2-5]. В недавней работе [6] было показано, что низкочастотная (частоты порядка $10 \mathrm{kHz}$ ) экранировка „грязной“ сверхпроводящей пленки (с большим удельным сопротивлением в нормальном состоянии) может вырасти в несколько раз при нанесении на нее тонкого слоя низкоомного нормального металла и магнитный отклик в основном определяется наведенной сверхпроводимостью в нормальном металле, что отличает такие структуры от других $S N$ систем с эффектом близости [4,5]. Кроме того, для такой $S N$ структуры с большим отношением удельных сопротивлений была предсказана сильно нелинейная и немонотонная зависимость сверхпроводящей плотности тока от сверхскорости (см. рис. 3, $a$ в [6]), что может приводить к особенностям нелинейного отклика такой гибридной структуры. Ранее для объяснения нелинейных эффектов в обычных сверхпроводниках были предложены различные механизмы нелинейности: нелинейность, связанная с подавлением модуля параметра порядка сверхтоком; с наличием джозефсоновских связей между гранулами в исследуемых образцах; с движением вихрей и с тепловой нелинейностью [7-16].

В нашей работе с помощью метода ближнепольной СВЧ микроскопии была исследована температурная зависимость мощности третьей гармоники структур сверхпроводник-нормальный металл MoN/Al c большим отношением удельных сопротивлений в зависимости от мощности СВЧ сигнала и постоянного магнитного поля. Обнаружено, что зависимость $P_{3 \omega}(T)$ может демонстрировать дополнительный (низкотемпературный) максимум и иметь нули при $T<T_{c}$, что ранее не наблюдалась в отклике на третьей гармонике в других сверхпроводящих системах. Эксперимент с магнитным полем говорит в пользу того, что обнаруженный эффект связан с наведенной, в слое нормального металла, сверхпроводимостью.

\section{2. Структуры сверхпроводник-нормальный металл}

В работе исследована серия двухслойных тонкопленочных гибридных структур сверхпроводник $(S)$-нормальный металл $(N)$, выращенных методом магнетронного напыления с базовым уровнем вакуума порядка $1.5 \cdot 10^{-7}$ mbar на стандартных кремниевых подложках размером $10 \times 10 \mathrm{~mm}^{2}$ без удаления оксидного слоя. Сначала напылялся Мо в атмосфере смеси газов $\mathrm{Ar}: \mathrm{N}_{2}=10: 1$ при давлении $1 \cdot 10^{-3}$ mbar и комнатной температуре, что позволяло получить однородные сверх- 
проводящие $\mathrm{MoN}$ пленки с критической температурой порядка $8 \mathrm{~K}$. Затем in-situ напылялся $\mathrm{Al}$ в атмосфере аргона при давлением $1 \cdot 10^{-3} \mathrm{mbar}$. Толщины слоев были измерены методом рентгеновской дифрактометрии. Толщина слоя нитрида молибдена была фиксирована и составляла величину $d_{\mathrm{MoN}} \cong 20 \mathrm{~nm}$, а толщина слоя алюминия $d_{\mathrm{Al}}$ варьировалась в диапазоне от 16 до $100 \mathrm{~nm}$. Значение величины удельного сопротивления сверхпроводящих структур при комнатной температуре составляло $\rho \cong 1.5-3 \mu \Omega \cdot \mathrm{cm}$ для $S N$ структур и $\rho \cong 100 \mu \Omega \cdot \mathrm{cm}$ для MoN. Значение плотности критического тока при температуре $T=4.2 \mathrm{~K}$ имеет величину $j_{c} \cong 10^{6} \mathrm{~A} / \mathrm{cm}^{2}$. Величина критической температуры для сверхпроводящих структур изменялась в диапазоне $T_{c}=7-8 \mathrm{~K}$.

\section{3. Экспериментальная установка}

В работе нелинейные СВЧ свойства $S N$ структур были исследованы с помощью метода ближнепольной СВЧ микроскопии. Этот метод основан на регистрации нелинейного СВЧ отклика с помощью зонда индуктивного типа (рис. 1) [17,18]. Зонд представляет собой тонкую медную проволочку диаметром порядка $50 \mu \mathrm{m}$, закорачивающую внутренний и внешний проводники коаксиального кабеля. Частота первой гармоники в эксперименте была равна $472 \mathrm{MHz}$. Максимальный уровень падающей мощности на частоте первой гармоники при измерениях был порядка $100 \mathrm{~mW}$. Для предотвращения контактной нелинейности между образцом и ближнепольным СВЧ зондом помещалась тефлоновая пленка. Этот метод позволяет создавать довольно сильное СВЧ поле, локализованное вблизи зонда на масштабах порядка его размеров $50 \mu \mathrm{m} \cdot 2 \mathrm{~mm}$. Мощность отраженного сигнала на частоте третьей гармоники $P_{3 \omega}$ излучаемая $S N$ структурой измерялась тем же самым зондом по которому подавался сигнал СВЧ на первой гармонике в зависимости от температуры, мощности СВЧ сигнала и перпендикулярного магнитного поля. Следует отметить, что к результатам с применением мощности $100 \mathrm{~mW}$ необходимо относится с осторожностью, поскольку данная мощность, как будет экспериментально показано ниже, вызывает локальный разогрев СВЧ зондом на величину порядка $0.5 \mathrm{~K}$. В то же время, такой нагрев отсутствует в случае малых мощностей порядка $1 \mathrm{~mW}$.

\section{4. Экспериментальные результаты}

На рис. 2 показана температурная зависимость мощности третьей гармоники структуры MoN/Al при различных СВЧ мощностях при толщине слоя алюминия $16 \mathrm{~nm}$. Ранее было показано, что температурная зависимость нелинейного СВЧ отклика сверхпроводящих пленок MoN имеет резкий максимум вблизи критической температуры перехода [19]. Как видно из

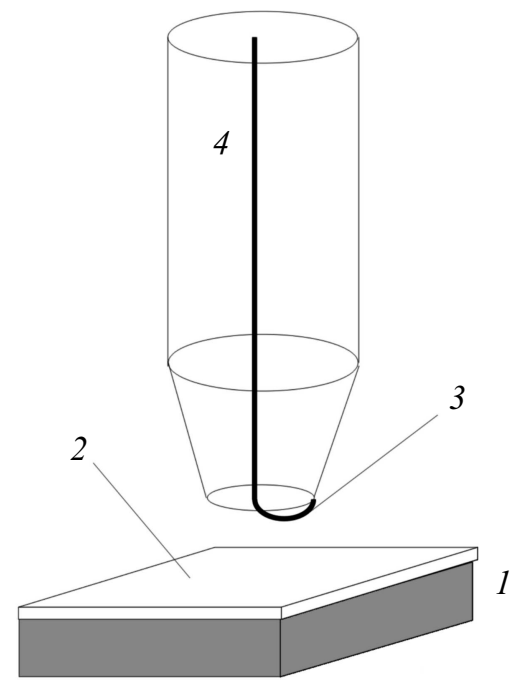

Рис. 1. Блок схема ближнепольного СВЧ зонда. $1-$ сверхпроводящая $S N$ структура, 2 - тефлоновая пленка, 3 - ближнепольный СВЧ зонд, 4 - коаксиальный кабель.

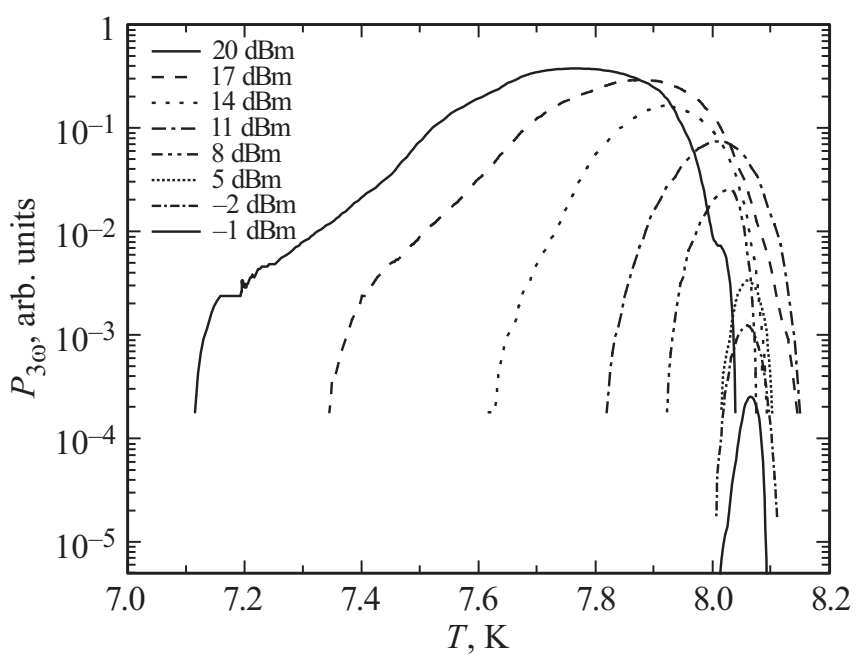

Рис. 2. Температурная зависимость мощности третьей гармоники при различных уровнях СВЧ мощности $S N$ структур MoN/Al. Толщина слоя алюминия составляет $16 \mathrm{~nm}$.

рис. 2, при малой толщине алюминия структуры наблюдается также один пик нелинейности вблизи $T_{c}$ и происходит слабый сдвиг критической температуры $\Delta T_{c} \cong 0.1 \mathrm{~K}$ по сравнению с пленкой $\mathrm{MoN}$, поскольку величина длины когерентности $\xi_{s}=6 \mathrm{~nm}$ для пленок MoN [6] много меньше ее толщины. Заметим, что СВЧ мощности порядка $100 \mathrm{~mW}$ приводят к локальному нагреву образца и сдвигу критической температуры на $0.5 \mathrm{~K}$ (это видно из сдвига зависимости $P_{3 \omega}(T)$ в низкие температуры).

На рис. 3 показана температурная зависимость $P_{3 \omega}(T)$ для структуры MoN/Al при толщине слоя алюминия $100 \mathrm{~nm}$. При увеличении толщины алюминия до $100 \mathrm{~nm}$ для структуры MoN/Al на $P_{3 \omega}(T)$ наблюдается 


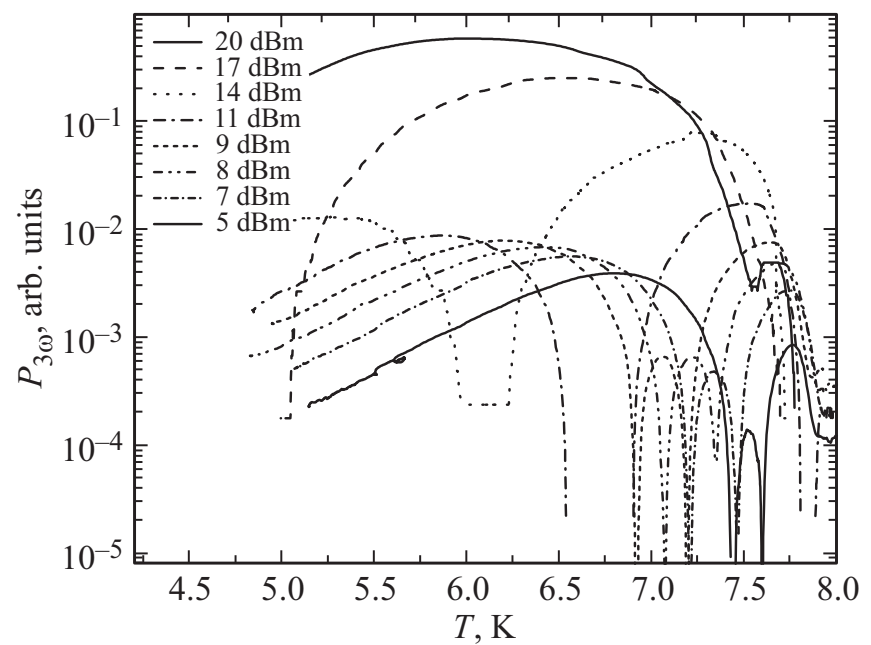

Рис. 3. Температурная зависимость мощности третьей гармоники при различных уровнях СВЧ мощности $S N$ структур MoN/Al. Толщина слоя алюминия составляет $100 \mathrm{~nm}$.

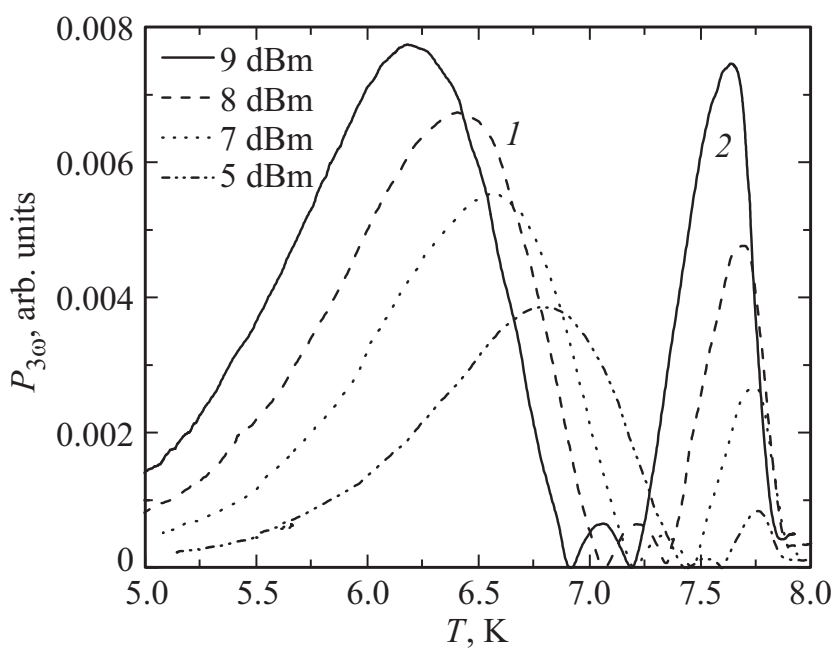

Рис. 4. Температурная зависимость мощности третьей гармоники при низких уровнях СВЧ мощности $S N$ структур MoN/A1. Толщина слоя алюминия составляет $100 \mathrm{~nm}$. Цифрами 1 и 2 показано положение дополнительного и основного максимума нелинейности, соответственно.

дополнительный низкотемпературный максимум нелинейности, который появляется при уменьшении мощности СВЧ сигнала. Наличие второго максимума на $P_{3 \omega}(T)$ в $S N$ структурах связано, по нашему мнению, с наведенной сверхпроводимостью в слое алюминия. Ранее линейные свойства аналогичных гибридных структур MoN/Al были изучены нами с помощью индуктивной методики [6]. В этих структурах наблюдался эффект близости, однако температурная зависимость магнитной восприимчивости демонстрировала один сверхпроводящий переход вблизи критической температуры.

Кроме появления второго пика при низких уровнях мощности СВЧ сигнала на зависимости $P_{3 \omega}(T)$ наблю- даются нули мощности третьей гармоники (см. рис. 4). Как известно, в сверхпроводниках нелинейный отклик возникает при переходе в сверхпроводящее состояние и отличен от нуля вплоть до низких температур [17-20]. В то же время, из наших наблюдений следует, что в случае $S N$ структур, существуют такие температуры ниже $T_{c}$ при которых нелинейный СВЧ отклик обращается в ноль. Это свойство $S N$ структур может быть интересно, например, с прикладной точки зрения, поскольку нелинейные эффекты в них могут быть значительно уменьшены при определенных температурах. В то же время следует отметить, что природа нулей мощности третьей гармоники в $S N$ структурах нам до конца не ясна и для ее объяснения необходимы детальные теоретические расчеты в рамках различных механизмов нелинейного СВЧ отклика.

Чтобы проверить, что второй пик на зависимости $P_{3 \omega}(T)$ связан с наведенной сверхпроводимостью в нормальном металле мы исследовали, как меняется $P_{3 \omega}(T)$ при наличии перпендикулярного магнитного поля. Действительно, эффективная длина когерентности в $N$ слое $\xi_{N} \sim\left(D_{N} / T\right)^{1 / 2} \sim 60 \mathrm{~nm}$ примерно в десять раз больше чем в $S$ слое $\left(D_{N}-\right.$ коэффициент диффузии в $N$ слое), что должно приводить к значительно меньшему значению второго критического поля $\sim \Phi_{0} / \xi_{N}^{2}\left(\Phi_{0}-\right.$ квант магнитного потока). Мы обнаружили, что амплитуда низкотемпературного максимума довольно сильно подавляется уже в полях порядка $50 \mathrm{G}$ (см. рис. 5), тогда как амплитуда максимума, расположенного вблизи $T_{c}$, уменьшается в этих полях незначительно. Кроме того, во внешнем магнитном поля амплитуда третьей гармоники изменяется более плавно от температуры и нули зависимости $P_{3 \omega}(T)$ исчезают (см. рис. 5). Таким образом можно сделать вывод, что нелинейный отклик вблизи $T_{c}$,

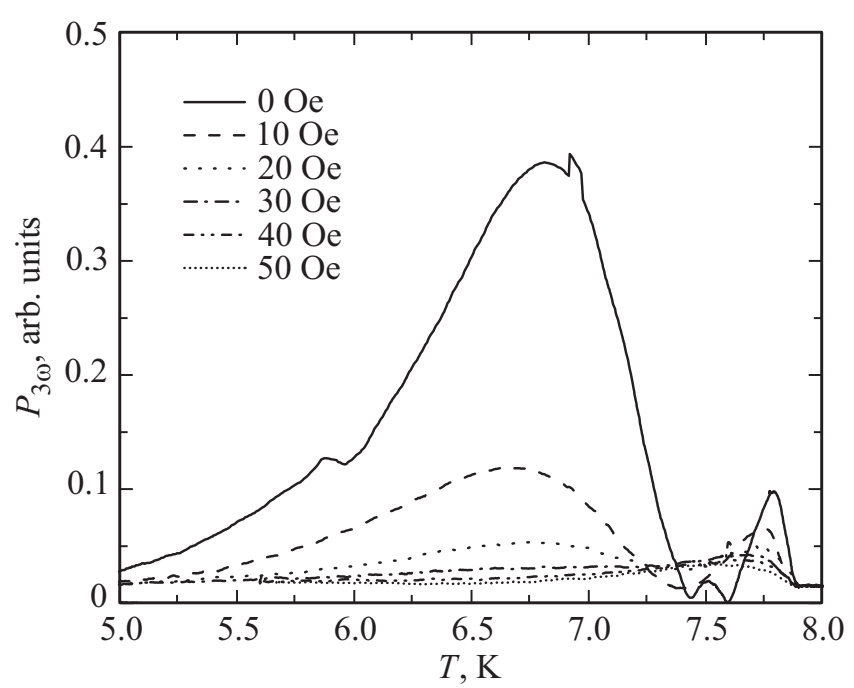

Рис. 5. Температурная зависимость мощности третьей гармоники в различных магнитных полях $S N$ структуры MoN/Al при толщине слоя алюминия $100 \mathrm{~nm}$. Уровень СВЧ мощности $P_{3 \omega}=5 \mathrm{dBm}$. 


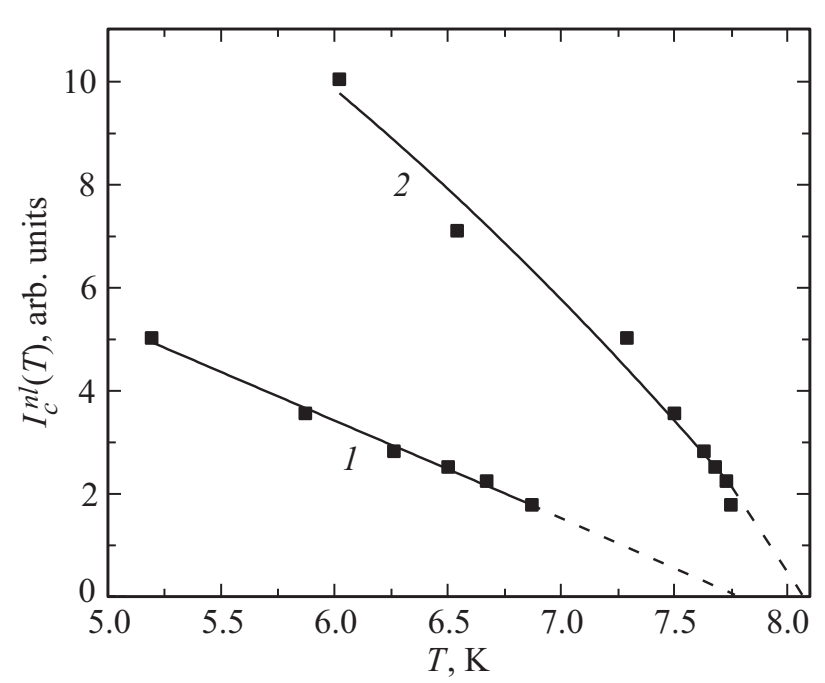

Рис. 6. Температурные зависимости критических токов, соответствующие дополнительному (1) и основному (2) максимуму нелинейности для $S N$ структуры MoN/Al толщиной $100 \mathrm{~nm}$.

обусловлен нелинейными свойствами пленки $\mathrm{MoN}$, a при более низких температурах связан с эффектом близости в слое нормального металла.

Из температурной зависимости $P_{3 \omega}(T)$ (см. рис. 3) структуры MoN/Al можно определить температурные зависимости критических токов нелинейности двух сверхпроводящих фаз [20]. Первая (низкотемпературная) фаза, как было отмечено выше, связана с наведенной сверхпроводимостью, а вторая (высокотемпературная) фаза обусловлена сверхпроводящими свойствами пленки MoN. Из теории известно, что максимум нелинейности достигается при условии, что СВЧ ток, текущий по сверхпроводнику, равен критическому току нелинейности $I_{\omega}=I_{c}^{n l}(T)$ [20]. На рис. 6 показаны температурные зависимости критических токов этих фаз. Из этих зависимостей можно определить, что величина критического тока сверхпроводящей фазы, связанной с наведенной сверхпроводимостью меньше величины критического тока, обусловленного сверхпроводником MoN. Bo-вторых, следует отметить, что первая и вторая фаза имеют близкие критические температуры.

В заключении следует отметить, что в качестве пробной гипотезы можно предположить, что на поверхности алюминия обычно присутствует слой $\mathrm{Al}_{2} \mathrm{O}_{3}$, который является барьером для сверхпроводящих пар. Поэтому, в принципе, может существовать два сверхпроводящих слоя, разделенным барьером. Эти слои могут иметь разные сверхпроводящие фазы, которые могут интерферировать в области между двумя максимумами. Кроме этого, подавление нелинейного СВЧ отклика слабым магнитным полем и малая величина критического тока дополнительного максимума нелинейности свидетельствует о наведенной сверхпроводимости в слое нормального металла.

\section{5. Заключение}

С помощью метода ближнепольной СВЧ микроскопии исследованы нелинейные СВЧ свойства структур сверхпроводник-нормальный металл MoN/Al при различных толщинах слоя нормального металла. В структурах MoN/Al обнаружен дополнительный (низкотемпературный) максимум в зависимости $P_{3 \omega}(T)$, связанный с наведенной сверхпроводимостью в слое нормального металла. Обнаружено зануление сигнала мощности третьей гармоники при низких уровнях мощности СВЧ сигнала при температурах ниже $T_{c}$ в структурах MoN/Al (с достаточно толстым слоем $\mathrm{Al}$ ).

\section{Финансирование работы}

Работа поддержана РНФ (грант № 15-12-10020, в области экспериментальных исследований нелинейных СВЧ свойств гибридных структур) и Программой фундаментальных исследований Президиума РАН „Фундаментальные проблемы высокотемпературной сверхпроводимости“. В работе использовано оборудование ЦКП „Физика и технология микро- и наноструктур“.

\section{Конфликт интересов}

Авторы заявляют, что у них нет конфликта интересов.

\section{Список литературы}

[1] P.G. de Gennes. Superconductivity of Metals and Alloys. Westview Press, Boulder, CO (1999).

[2] S.V. Lempitskii. Physica C 167, 168 (1990).

[3] T. Bergmann, R.H. Kuhl, B. Schröder, M. Jutzler, F. Pobell. J. Low Temp. Phys. 66, 209 (1987).

[4] A.C. Mota, P. Visani, A. Pollini. Low Temp. Phys. 76, 465 (1989).

[5] A.D. Zaikin. Solid State Commun. 41, 533 (1982).

[6] D.Yu. Vodolazov, A.Yu. Aladyshkin, E.E. Pestov, S.N. Vdovichev, S.S. Ustavshikov, M.Yu. Levichev, A.V. Putilov, P.A. Yunin, A.I. El'kina, N.N. Bukharov, A.M. Klushin. Supercond. Sci. Technol. 31, 115004 (2018).

[7] Л.П. Горьков, Г.М. Элиашберг. ЖЭТФ 54, 612 (1968).

[8] O. Entin-Wohlman. Phys. Rev. B 18, 4762 (1978).

[9] T. Jujo. J. Phys. Soc. Jpn. 87, 024704 (2018).

[10] B.A. Willemsen, K.E. Kihlstrom, T. Dahm, D.J. Scalapino, B. Gowe, D.A. Bonn, W.N. Hardy. Phys. Rev. B 58, 6650 (1998).

[11] В.В. Курин, А.А. Уткин. ЖЭТФ 127, 652 (2005).

[12] T. Dahm, D.J. Scalapino. Appl. Phys. Lett. 85, 4436 (2004).

[13] J. Halbritter. J. Supercond. 8, 691 (1995).

[14] А.И. Ларкин, Ю.Н. Овчинников. ЖЭТФ 68, 1915 (1975).

[15] M.A. Hein. High-Temperature Superconductor. Thin Films at Microwave Frequencies. Springer, Berlin (1999). 394 p. 
[16] T.M. Mishonov, N. Cheenne, D. Robbes, J.O. Indekeu. Eur. Phys. J. B 26, 291 (2002).

[17] E.E. Pestov, Yu.N. Nozdrin, V.V. Kurin. IEEE Trans. Appl. Supercond. 11, 131 (2001).

[18] А.Ю. Аладышкин, А.А. Андронов, Е.Е. Пестов, Ю.Н. Ноздрин, В.В. Курин, А.M. Cucolo, R. Monaco, М. Boffa. Изв. вузов. Радиофизика 46, 123 (2003).

[19] Yu. Aladyshkin, A.Yu. Klimov, M.A. Kucherenko, E.E. Pestov, V.V. Kurin, Yu.N. Nozdrin, V.L. Mironov. Mod. Phys. Lett. B 17. 627 (2003).

[20] S.V. Baryshev, E.E. Pestov, A.V. Bobyl, Yu.N. Nozdrin, V.V. Kurin. Phys. Rev. B 76, 054520 (2007).

Редактор Д.В. Жуманов 